\title{
La croissance et le développement corporel de la naissance à la maturité dans la race ovine iranienne Mehraban à queue grasse
}

\author{
S.S. Bathaei ${ }^{1}$
}

BATHAEI (S.S.).La croissance et de développement corporel de la naissance à la maturité dans la race ovine iranienne Mehraban à queue grasse. Revue Élev. Méd. vét. Pays trop., 1995, 48 (2) : 181-194

La croissance de 15 mesures corporelles linéaires dans la race ovine iranienne Mehraban à queue grasse a été analysée par la méthode des moindres carrés. Les mesures concernant 1238 animaux ont été prises à intervalles de 3 mois, entre 3 et 48 mois d'âge de 1984 à 1990 . Les effets du sexe, du mode de naissance, de l'année de naissance et de l'âge de la mère sur le poids et sur les 15 caractères mesurés ont été étudiés. Le sexe est le seul facteur dont l'influence soit significative sur tous les caractères aux différents âges. Les courbes de croissance ont été établies avec précision à partir des valeurs moyennes à 16 âges différents. L'estimation à 98 p. 100 du degré de maturité est, pour les mâles et les femelles, de 25 et 22 mois respectivement. L'application de l'allométrie aux caractères mesurés a été examinée en vue d'établir des courbes de croissance plus précises. La taille au garrot, la longueur du corps, le périmètre abdominal et le périmètre thoracique manifestent le mieux les variations du poids et parmi ces caractères, le périmètre thoracique est le meilleur indicateur de ces variations aux différents âges. Quant aux autres facteurs, ils évoluent presque indépendamment du poids.

Mots clés: Ovin Meharaban - Croissance - Gain de poids - Maturité Mensuration corporelle - Iran.

\section{INTRODUCTION}

En Iran, la majorité des cheptels ovin et caprin sont élevés selon le système de la production extensive. Les animaux se nourrissent essentiellement sur des pâturages naturels dont la superficie atteint environ 100 millions d'hectares, soit 58 p. 100 de la superficie totale du pays. La plupart sont cependant médiocres, tant du point de vue de la qualité que de la quantité, et seulement 10 millions d'hectares produisent $450 \mathrm{~kg}$ de matière sèche/ha/an. Dans ces conditions, la production annuelle des pâturages permet de nourrir environ 16 millions de moutons et de chèvres alors qu'on estime actuellement leur nombre à plus de 60 millions. Pour résoudre ce problème, il est indispensable de réduire le nombre d'animaux peu productifs et de sélectionner un bon format en fonction de la disponibilité alimentaire et d'autres facteurs comme le mode d'élevage.

La présente étude a été menée sur la race ovine Mehraban à queue grasse, pour déterminer à quel âge la maturité est atteinte et avoir plus de données sur le dévelop-

1. Université Libre de Bruxelles, Section Interfacultaire d'Agronomie, Av. Paul Hèger 28, C.P.169, 1050 Bruxelles, Belgique.

Reçu le 21.12.1994, accepté le 15.6.1995. pement, la modification de forme et les changements corporels en fonction du temps. Selon Prud'hon (21), la prise en considération du degré de maturité aura un intérêt réel dans la mesure où l'on connaîtra bien les lois du développement relatif des principaux organes et tissus au cours de la croissance. Par ailleurs, la connaissance de la maturité permettra de choisir le format optimal pour un programme de sélection ultérieure et d'établir des comparaisons avec d'autres races ovines. Cette étude permet aussi d'établir les relations existant entre format et poids, de définir le meilleur indicateur pondéral parmi les caractères mesurés, ce qui sur le plan pratique facilitera la mesure sur les animaux.

\section{MATÉRIEL ET MÉTHODES}

La race ovine iranienne Mehraban à queue grasse est une race à viande, rustique et bien adaptée au milieu semiaride où elle vit depuis des siècles (1). Son berceau se trouve dans la province de Hamadan située au nord-ouest de l'Iran où elle est élevée de manière semi-extensive. Elle figure parmi les races sédentaires et constitue 4 à 5 p. 100 du cheptel national avec 1200000 têtes environ en 1994.

Les animaux utilisés ici proviennent d'un troupeau élevé dans cette région. Ils sont restés 7 mois par an en pâturage. A la fin de chaque été, en raison de la médiocrité de l'alimentation, ils ont reçu un supplément composé d'orge et de luzerne puis, étant donné la rigueur des hivers, ils ont été nourris en bergerie pendant les 5 mois suivants avec une ration composée de céréales et de luzerne.

Qinze caractères ont été mesurés afin de définir le format et l'âge de maturité dans cette race. Le développement peut être défini comme le changement de la composition corporelle durant la croissance de l'animal. 'II peut être influencé par différents facteurs parmi lesquels le taux de croissance individuel, le sexe, le mode de naissance, l'année de naissance et l'âge de la mère à l'agnelage. Ces facteurs ont été étudiés. Les instruments utilisés ont été un mètre à ruban flexible et une toise, tous deux gradués en centimètres. Les caractères mesurés sont illustrés dans la figure 1.

Les données enregistrées entre 1984 et 1990, concernant 1238 moutons de la race Mehraban âgés de 3 à 48 mois, ont été analysées par la méthode des moindres carrés (13). Après ajustement des données, les animaux ont été classés en 16 groupes d'âge de 3 à 48 mois, par intervalles de 3 mois, pour réduire les variations du poids 
S.S. Bathaei
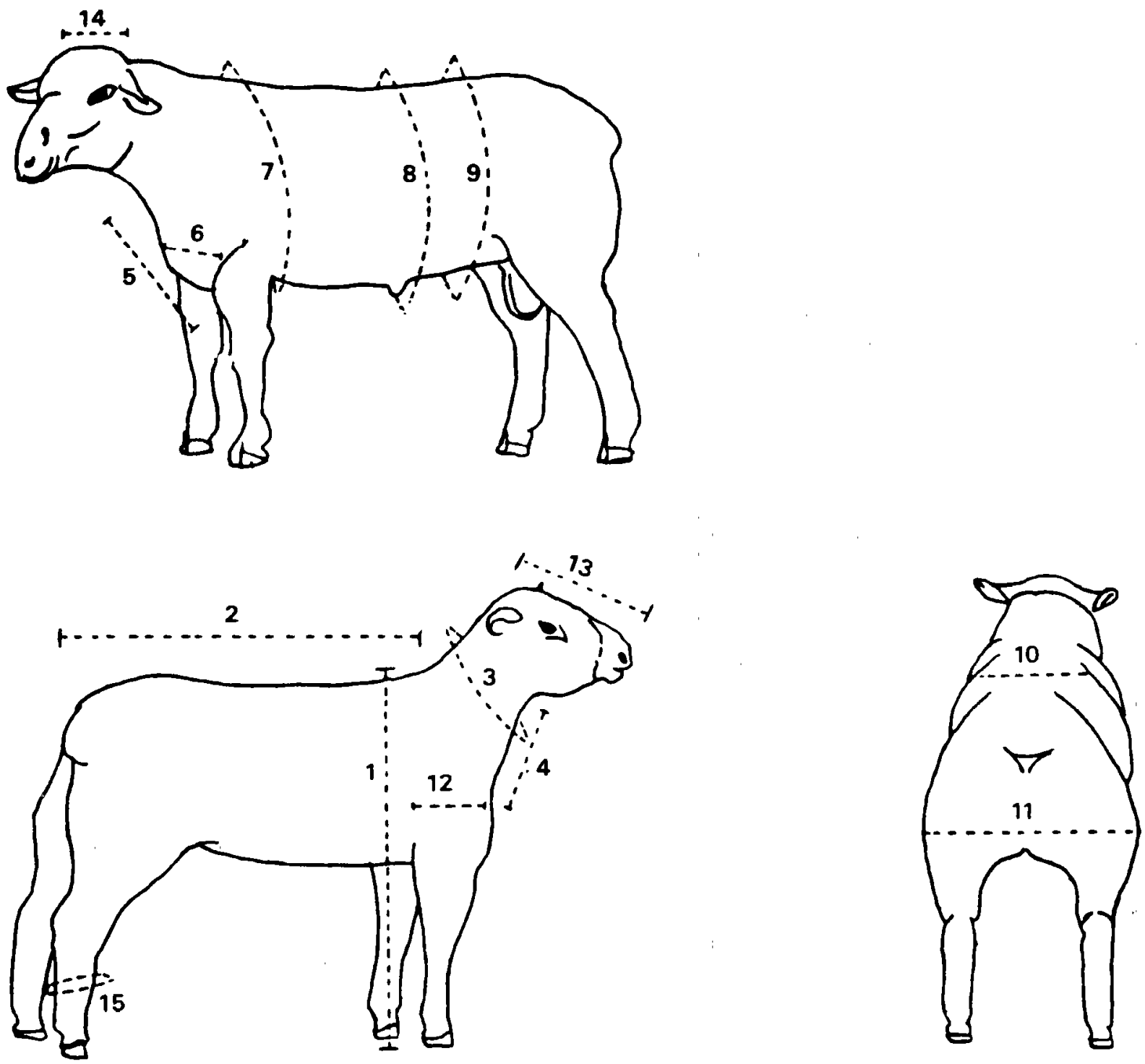

Figure 1 : Caractères mesurés.

1. Taille au garrot ; 2. longueur du corps ; 3. pourtour du cou; 4. longueur du cou; 5. longueur de la poitrine; 6. largeur de la poitrine; 7. périmètre thoracique ; 8. pourtour du corps ;9. périmètre abdominal ; 10. largeur du dos; 11 . largeur du gigot; 12 . largeur de l'épaule ; 13 . longueur de la tête; 14. largeur de la tête; 15 . périmètre du canon.

et des caractères mesurés imposées par les variations saisonnières. Le modèle linéaire suivant a été utilisé pour les variables observées:

$\mathrm{Y}_{\mathrm{ijklm}}=\mu+s_{i}+a_{j}+t_{k}+m_{i}+e_{i j k l m}$

dans lequel : $Y_{i j k m m}=$ poids, taille au garrot, longueur du corps, pourtour du cou, longueur du cou et de la poitrine, largeur de la poitrine, périmètre thoracique, pourtour du corps, périmètre abdominal, largeur du dos, du gigot, de l'épaule, longueur de la tête, largeur de la tête et périmètre du canon de l'animal $m$, de sexe $i$, de mode de naissance $j$, né l'année $k$ et de l'âge de la mère $/$; $\mu=$ moyenne générale ;

$\mathrm{s}_{\mathrm{i}}=$ effet fixe du sexe $\quad i=(1=$ mâle, 2 = femelle $)$;

$\mathrm{a}_{\mathrm{i}}=$ effet fixe du mode de naissance $\mathrm{j}=(1=$ simple, 2 = double) ;

$\mathrm{t}_{\mathrm{k}}=$ effet fixe de l'année de naissance $\mathrm{k}=(1,2, \ldots, 7)$ correspondant à 7 années (1984-1990) ;

$m_{1}=$ effet fixe de l'âge de la mère à l'agnelage

$1=$

$(1,2, \ldots, 6)$ correspondant à 6 classes d'âge, de 1 à 6 ans ;

$e_{\mathrm{ijklm}}=$ effet résiduel aléatoire. 
L'équation de Brody (4) a été utilisée pour établir une courbe asymptotique exponentielle, pour le poids et les 15 caractères mesurés, soit :

$Y_{\mathrm{t}}=A\left[1-e^{-k\left(1-t^{*}\right)}\right]$

où : $Y$, est la mesure du caractère envisagé à l'âge $t, A$ est la valeur finale du caractère mesuré, $k$ est une constante correspondant au taux relatif de croissance, $t$ est l'âge correspondant à une valeur donnée de $Y$ et $t^{*}$ est une constante qui représente l'origine du temps de la courbe (ici la naissance).

Cette équation peut être utilisée sous une autre forme (26) pour définir le degré de maturité à un âge donné. Dans ce cas, elle se présente ainsi :

$Y_{t}=A\left[1-\left(1-u_{0}\right) \cdot e(-t / \tau)\right]$

A représente la valeur à la maturité du caractère envisagé, $u_{0}$ est la proportion de maturité à la naissance $(t=0)$, et $\tau=$ $1 / \mathrm{k}$ est un temps constant ou l'intervalie de maturité.

Les équations [2] et [3] ont été utilisées afin de définir le degré de maturité $Y$ pour les différents caractères à différents âges et pour les deux sexes. Le degré de maturité a été calculé par $u_{t}=Y_{\mathrm{t}} / A$. Il est présenté en pourcentage.

Les mesures du corps sont souvent examinées en termes d'allométrie, c'est-à-dire en termes de croissance relative $(4,26,30)$ d'un organe comparée généralement au poids total ou à tout autre système de référence (Taylor, communication personnelle). La relation d'allométrie est l'instrument indispensable de toute étude de la croissance relative et elle exprime les changements de proportions.

La relation d'allométrie, $Y=a X^{b}$, peut être représentée sous la forme logarithmique (23) :

$\log Y=\log a+b \log X$

Deux paramètres définissent cette relation : $b$ est le coefficient d'allométrie et a représente la valeur de $Y$ lorsque $X=I$ est l'indice d'origine. Cette relation a été utilisée pour décrire plus efficacement les courbes de croissance pour les caractères mesurés.

Les principales sources de variations du poids ainsi que l'importance relative de chaque caractère ont été calculées en utilisant la régression multiple. Les équations de régression ont été calculées par étapes selon une procédure (27) qui donne un coefficient maximum de détermination multiple $\left(R^{2}\right)$. A chaque étape, une variable a été ajoutée à l'équation. La variable ajoutée était celle qui réduirait au maximum la somme des carrés des erreurs ou celle qui aurait la plus grande corrélation partielle avec la variable dépendante (ici le poids), parmi les variables non encore incluses. Deux restrictions ont été apportées à cette procédure. Premièrement, pour qu'une variable soit ajoutée à l'équation, sa réduction de l'erreur de la somme des carrés doit être suffisamment élevée pour que la valeur de $\mathrm{F}$ soit supérieure ou égale à la première variable. Deuxièmement, si après avoir ajouté les variables, la valeur de $F$ pour une d'entre elles introduite lors d'une étape précédente devient moindre que la précédente, cette variable est supprimée. Une description détaillée des procédures de régression par étapes a été donnée par Cody et Smith (5).

\section{RÉSULTATS}

Les résultats de l'analyse de variance du modèle linéaire [1] montrent que tous les effets sont significatifs $(p<0,001$ et $p<0,05)$ pour le poids. Ils confirment les études antérieures $(1,2)$. Tous les caractères mesurés sont significativement $(p<0,001$ et $p<0,05)$ influencés par l'effet du sexe (tableau I) à tous les âges. En revanche, le mode de naissance a un effet significatif ( $p<0,05$ ) seulement sur le poids, la taille au garrot, la longueur du corps, le pourtour du cou et le périmètre thoracique. L'année de naissance n'influence que le poids et le périmètre thoracique. L'âge de la mère à l'agnelage a un effet significatif $(p<0,05)$ sur le poids, la taille au garrot et la longueur du corps seulement jusqu'au sevrage (90 jours).

En ce qui concerne le coefficient de détermination $\left(R^{2}\right)$ du modèle, plus de 90 p. 100 de la variation totale est expliqué pour les caractères suivants : taille au garrot, longueur du corps, pourtour du cou, périmètre thoracique, pourtour du corps et périmètre abdominal. Entre 70 et 80 p. 100 est expliqué pour la longueur et la largeur de la tête, la longueur et la largeur de la poitrine, la largeur du dos, la largeur du gigot, la largeur de l'épaule et la longueur du cou. Quarante huit p. 100 seulement de la variation est expliqué par le modèle pour le périmètre du canon (tabl. I). Les moyennes des 15 caractères mesurés ajustées par la méthode des moindres carrés pour le sexe, le type de naissance, l'année de naissance et l'âge de la mère sont données dans le tableau II.

\section{Développement corporel}

Les estimations des paramètres de courbes de croissance obtenues à partir des équations [2] et [3] en vue d'analyser le développement corporel, pour le poids et les 15 caractères pour le mâle et la femelle, sont données au tableau III. Les caractéristiques des courbes de croissance (tabl. III) obtenues par l'équation de Brody (4) ont été utilisées pour établir les courbes de croissance des 15 caractères mesurés séparément pour les mâles et les femelles. (fig. 2, 3).

En ce qui concerne la proportion de la maturité à la naissance ( $u_{0}$ dans l'équation 3 ), les caractères précoces à la naissance sont le périmètre du canon, la longueur du cou, la longueur et la largeur de poitrine, la taille au garrot et la largeur du gigot. Ils ont à la naissance entre 55 et 70 p. 100 de leur maturité finale, et ont un intervalle de maturité de 5 à 8 mois tandis que les autres caractères parmi les 15 étudiés ici ont un intervalle de maturité com- 
TABLEAU I

Analyses de variance du modèle linéaire $(l)$ pour les effets du sexe, du mode de naissance, de l'année de naissance et de l'âge de la mère sur le poids et les 15 caractères mesurés de 3 à $\mathbf{4 8}$ mois dans la race Mehraban

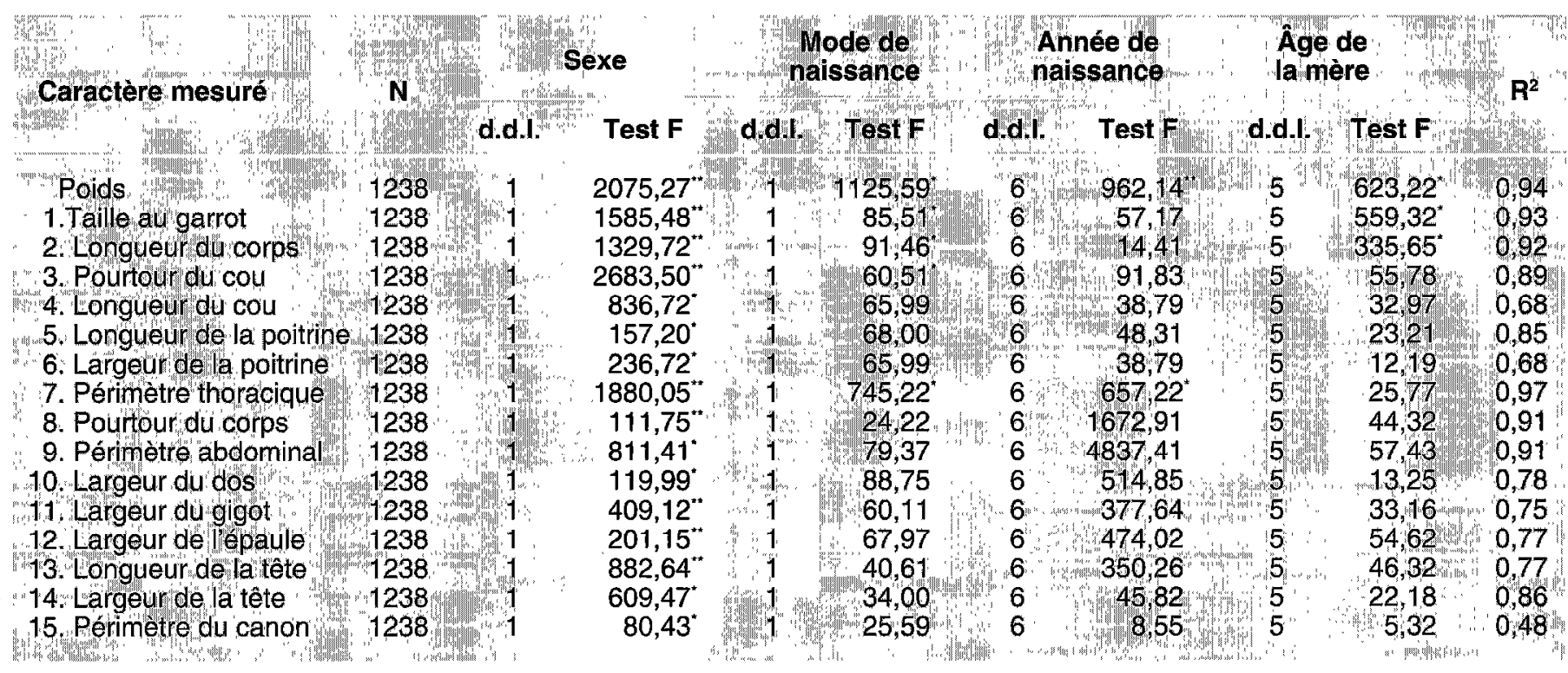

$p<0,05 ; " p<0,001$.

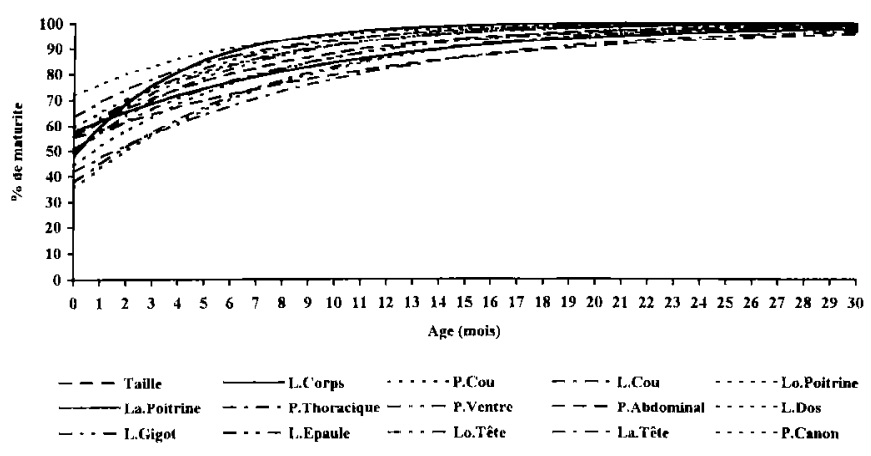

Figure 2 : Courbes de croissance des 15 caractères comme pourcentage. de maturité pour le sexe mâle dans la race Mehraban.

pris entre 8 et 12 mois. Le périmètre du canon atteint plus de 90 p. 100 de la maturité finale avant 6 mois.

La résiduelle de déviation standard dans le tableau III, en ce qui concerne le pourcentage de la maturité, montre une courbe asymptotique exponentielle, généralement mieux tracée pour les caractères de maturité précoce, car il y a moins de déviation. La valeur générale de la présente hypothèse de croissance est renforcée par le fait que presque toutes les déviations atteignent moins de 1 p. 100 de la maturité au moment où celle-ci atteint 98 p. 100.

Les mesures corporelles linéaires dans la croissance des ovins, quand elles sont exprimées en pourcentage de leur valeur adulte, montrent clairement que certains caractères corporels sont moins matures que d'autres à tout moment avant la maturité. Ils peuvent être classés et ce classement, à quelques exceptions près, reste inchan-

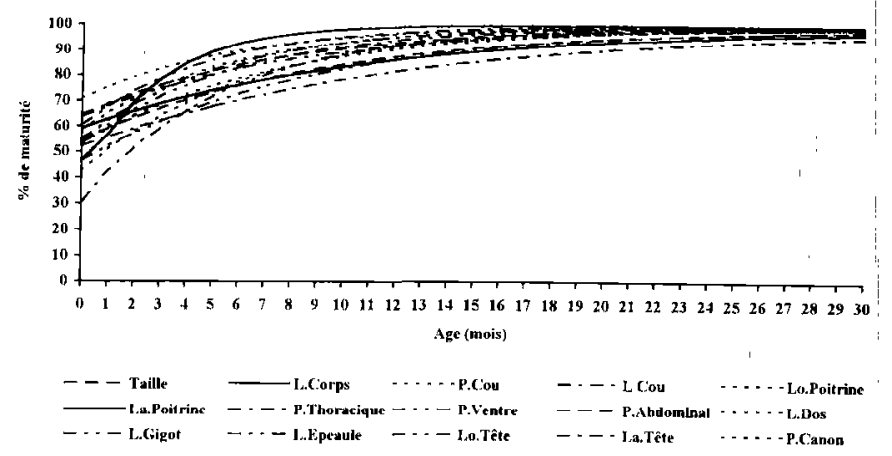

Figure 3 : Courbes de croissance des 15 caractères comme pourcentage de maturité pour le sexe femelle dans la race Mehraban.

gé durant toute la croissance. Dans cette perspective, les 15 caractères sont regroupés en 5 groupes de 3 en fonction de leur taux de maturité presque similaire. Les caractéristiques de chaque groupe (par sexe) figurent au tableau IV. II faut noter que l'ajustement de la courbe de Brody aux données fournit un paramètre de temps exponentiel pour chaque mesure corporelle, et que le résultat, en ce qui concerne la précocité de la maturité, correspond presque à celui qui est obtenu de façon empirique.

Les résultats indiqués dans le tableau IV montrent que, en prenant la moyenne des 15 caractères mesurés, les mâles arrivent à 95 p. 100 de leur maturité corporelle à l'âge de 18 mois, tandis que les femelles y parviennent dès 15 mois. De même, celles-ci parviennent plus rapidement que les mâles à 98 p. 100 de leur maturité : à 22 mois contre 25 pour ces derniers. Dans les 5 groupes, il existe une différence de 1 à 3 mois entre mâles et 
TABleaU II

Moyennes du poids (kg) et des 15 caractères mesurés $(\mathrm{cm})$ de 3 à 48 mois avec l'intervalle de 3 mois, ajustés pour le sexe, le mode de naissance, l'année de naissance et l'âge de la mère, dans la race Mehrahan

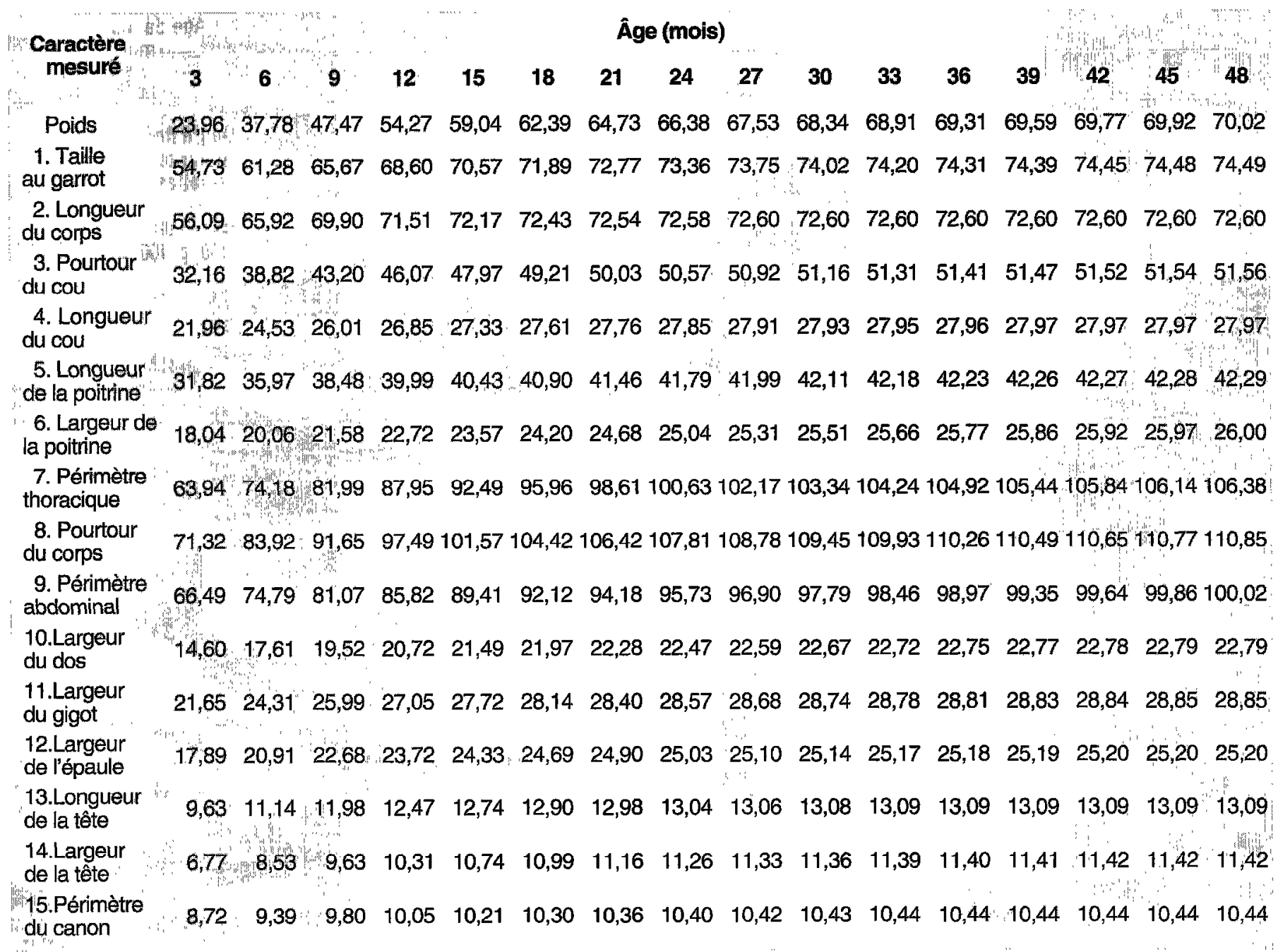
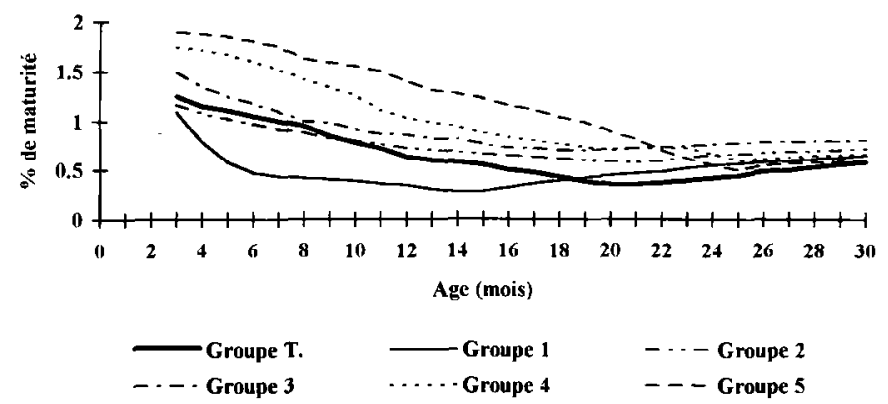

Figure 4 : Déviations des moyennes obtenues par la méthode des moindres carrés des courbes de croissance pour les 5 groupes ainsi que pour le groupe total des 15 caractères mesurés (pourcentage de format de maturité) pour le sexe mâle dans la race Mehraban.

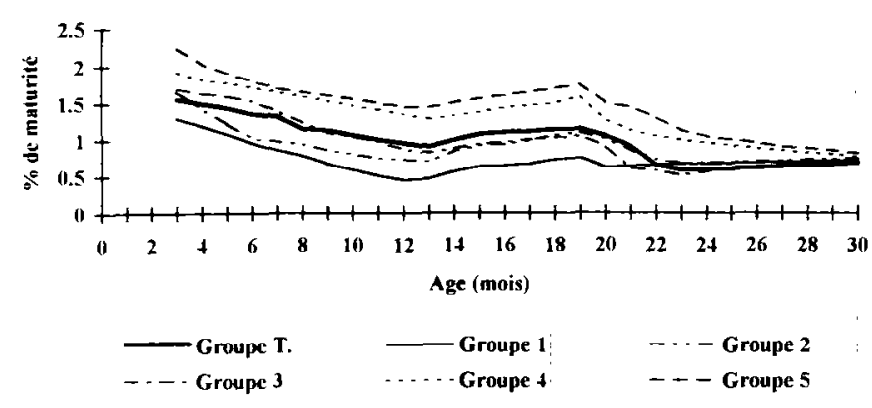

Figure 5 : Déviations des movennes obtenues par la méthode des moindres carrés des courbes de croissance pour les 5 groupes ainsi que pour le groupe total des 15 caractères mesurés (pourcentage de format dematurité) pour le sexe femelle dans la race Mehraban. 
TABLEAU III

Les caractéristiques des courbes de croissance pour le poids (kg) et les 15 caractères mesurés (cm) pour les femelles et les mâles dans la race Mehraban

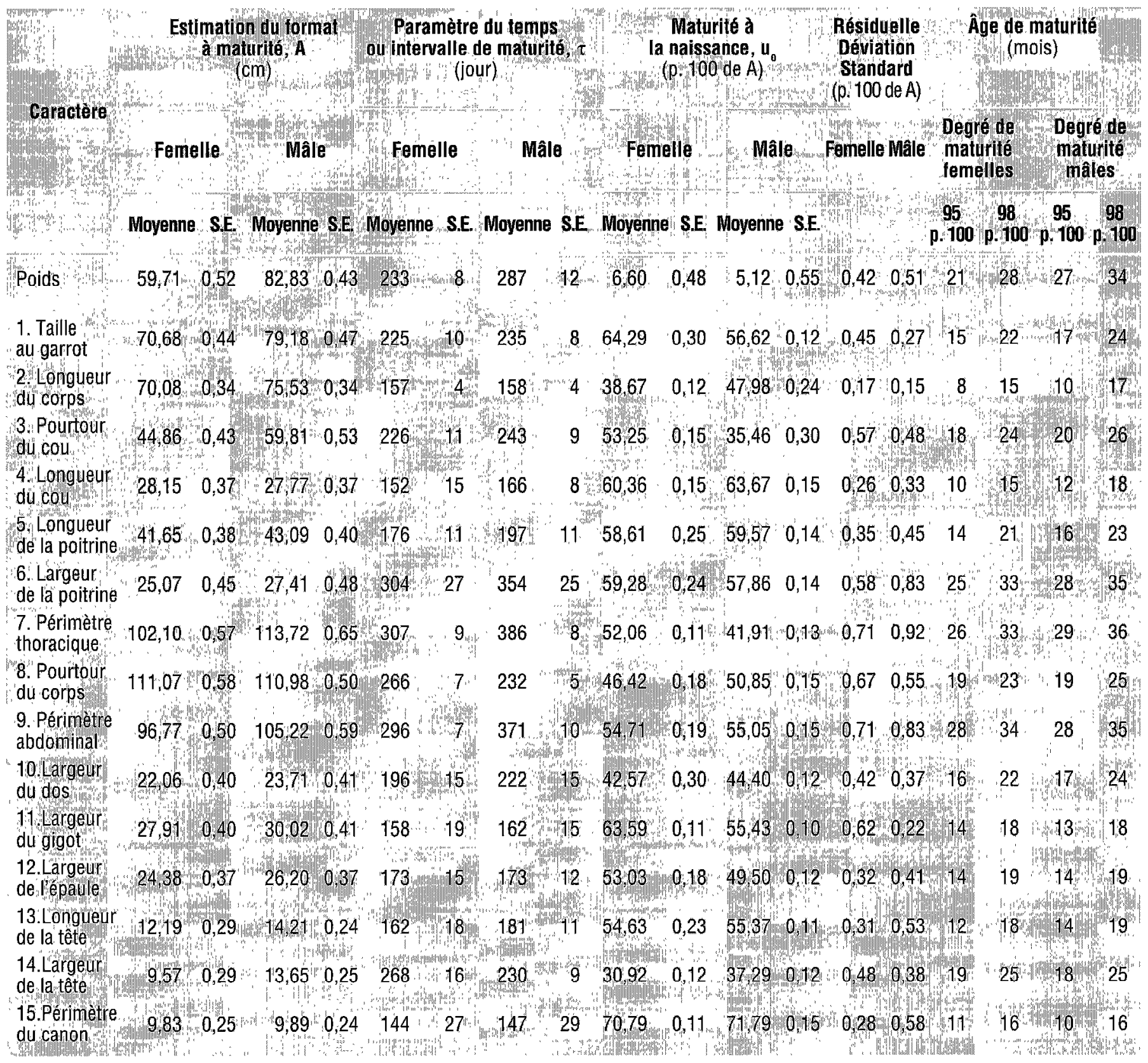

femelles : les femelles atteignent la maturité pour tous les caractères en une période plus courte que les mâles. Cela confirme la précocité des femelles.

En général, un animal sera précoce pour un caractère s'il atteint un degré spécifié de maturité dans un laps de temps inférieur à la moyenne. Par exemple, si le temps moyen pour l'ensemble des caractères est de 18 et 15 mois, respectivement pour les mâles et les femelles, les caractères qui font partie des groupes 1,2 et 3 , en particulier la longueur du corps, la largeur du gigot et la taille au garrot, sont considérés comme précoces et ceux qui font partie des groupes 4 et 5 , notamment les périmètres abdominal et thoracique, sont considérés comme tardifs.

Les déviations des moyennes obtenues par la méthode des moindres carrés pour les 5 groupes et le groupe total ont été utilisées (en pourcentage du format de maturité) 
TABLEAU IV

Âge de maturité (en mois) des différents groupes de caractères pour les deux sexes dans la race Mehraban

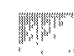 Groupes de caractères}

Groupe 1

(Longueur du corps, Longueur du cou, Périmètre du canon)

Groupe 2

(Longueur de la tête, Largeur du gigot, Largeur de l'épaule)

Groupe 3

(Taille au garrot, Longueur de la poitrine, Largeur du dos)

Groupe 4

(Pourtour du cou, Pourtour du corps, Largeur de la tête)

Groupe 5

(Périmètre thoracique, Périmètre abdominal, Largeur de la poitrine)

Moyenne des 16 caractères mesurés

pour étudier les changements de ces déviations avec l'âge (fig. 4, 5). Chez les mâles, les déviations diminuent rapidement avec l'âge dans les 5 groupes jusqu à l'âge de 20 mois, passant de 1-2 p. 100 de la maturité à 10,5 p. 100 selon le groupe. Après cet âge une légère hausse apparaît mais elle est moins importante, de l'ordre de 1 p 100 de la maturité (figure 4). Ce phénomène peut être dû à différents facteurs d'environnement ou à des caractéristiques individuelles, puisque la plupart des caractères ont déjà atteint la maturité. En ce qui concerne les femelles, la déviation diminue jusqu'à l'âge de 13 mois environ, de $1,5-2,5$ p. 100 à $0,5-1,5$ p. 100 selon le groupe, puis augmente entre 13 et 19 mois. Le fait que cette période coïncide avec la gestation, la mise bas et la lactation pourrait expliquer cette augmentation (fig. 5). Dans les 5 groupes, la variation des caractères diminue avec l'âge, et elle est moins élevée dans les groupes à maturité précoce que dans les groupes à maturité tardive. Ce phénomène peut être dû au fait que, la maturité y étant plus rapidement atteinte, les facteurs de l'environnement ont nettement moins d'influence que dans les groupes à maturité tardive.

\section{Relation d'allométrie}

Afin de comparer le rythme de développement d'un caractère à celui du corps, on a calculé le coefficient d'allométrie entre les pourcentages moyens de maturité de chacun des 5 groupes et le pourcentage de maturité total (moyenne des pourcentages de maturité des 15 caractères) de la naissance à 30 mois. Deux courbes allométriques basées sur les données de maturité qui ont été illustrées dans le tableau $\mathrm{V}$, sont présentées dans les figures 6 et 7 .

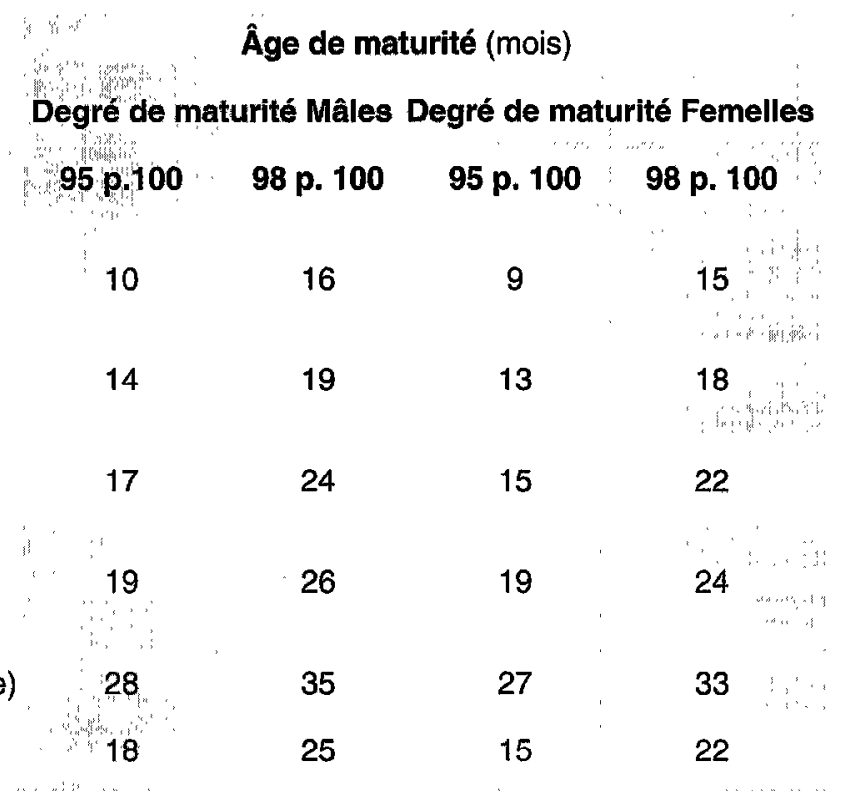

TABLEAU V

Relation des cinq groupes de caractères avec le groupe total par sexe

\begin{tabular}{rrrcrcr} 
& \multicolumn{3}{c}{ Mâle } & \multicolumn{3}{c}{ Femelle } \\
Groupe & & & & & \\
a & b & P & a & b & P \\
Groupe 1 & $-1,1381$ & 1,24 & 0,001 & $-0,4541$ & 1,12 & 0,001 \\
Groupe 2 & $-0,6123$ & 1,13 & 0,001 & $-0,5681$ & 1,09 & 0,001 \\
Groupe 3 & $-0,1095$ & 1,03 & 0,001 & $-0,2809$ & 1,06 & 0,001 \\
Groupe 4 & 0,3111 & 0,94 & 0,001 & 0,1208 & 0,98 & 0,001 \\
Groupe 5 & 1,2433 & 0,72 & 0,001 & 1,1693 & 0,75 & 0,001
\end{tabular}

$\mathrm{a}=$ indice d'origine

$b=$ coefficient d'allométrie

$P=$ test de signification

Le logarithme du degré de maturité, Y (équation [3]), pour chacun des cinq groupes de trois caractères qui présentaient un taux de maturité presque similaire, est comparé au logarithme du total des 15 caractères mesurés. Les points tracés sont dérivés des moyennes de moindres carrés linéaires. Les intercepts des droites en pointillé sur les axes à maturité dans les figures 6 et 7 montrent que les erreurs attendues dans l'estimation par allométrie du format à maturité sont assez faibles dans les 5 groupes et qu'elles sont toutes proches de la maturité. Les courbes qui relient les points s'incurvent progressivement sans se rejoindre et sont extrapolées jusqu'à l'axe horizontal ou vertical qui représente la complète maturité. Sachant qu'un intervalle de 0,1 sur cette échelle logarithmique correspond à 10 p. 100 de la maturité, il est possible d'observer les erreurs de l'estimation de maturité par allo- 
Degré moyen de maturité /groupe (Log)

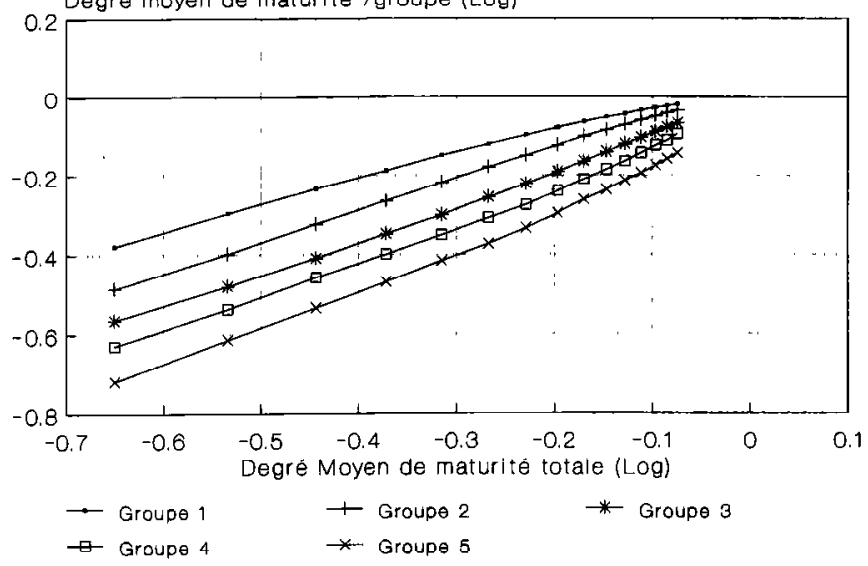

Figure 6 : Courbe allométrique du degré moyen de maturité des différents groupes chez les males dans la race Mehraban.

métrie d'une part, et d'autre part d'estimer ou prédire une moyenne de taux de maturité à partir d'un des groupes successifs. En effet, la différence de maturité entre deux groupes successifs varie entre 2 et 5 p. 100 (figures 6 et 7) et elle peut atteindre environ 20 p. 100 entre les deux groupes extrêmes (précoce ou tardif). La différence prévisible entre les caractères à l'intérieur d'un groupe est comparativement faible, de 2 à 3 p. 100 maximum, de même que les erreurs observées. Dans ce cas, l'allométrie peut être considérée comme suffisamment précise.

A partir d'une équation d'allométrie du type :

$Y=a X^{b}$ ou $\log Y=\log A+b \log X$

les coefficients (b) de croissance varient de 0,73 (groupe 5 , maturité tardive) à 1,24 (groupe 1 , maturité précoce) pour le sexe mâle et de 0,75 (groupe 5) à 1,09 (groupe 1) pour le sexe femelle (tabl. V). Le coefficient b (ou coefficient d'allométrie) est d'autant plus élevé que le caractère correspond à une croissance relative plus importante. $\mathrm{Ce}$ coefficient varie selon le caractère concerné et pour un même caractère, selon le sexe de l'animal. D'après Russell (26), les taux de croissance relatifs des différentes mesures corporelles ne peuvent pas être constants au cours de la croissance et une simple équation allométrique ne donnera pas une description non biaisée de croissance relative, à moins que les données soient réduites à une courte période ou que les caractères parviennent à maturité en même temps. C'est pourquoi on a mesuré, d'une part les caractères dans un intervalle régulier de 3 mois et, d'autre part, dans l'étape suivante, regroupé les caractères qui arrivent à maturité presque en même temps.

\section{Principales sources de variation du poids}

Si l'on compare le temps nécessaire pour arriver au poids adulte, en utilisant l'équation de Brody (4), au temps nécessaire pour atteindre la maturité corporelle (moyenne des pourcentages de maturité des 15 caractères), un certain écart apparaît : alors que cette durée s'élève respectivement à 21 et 28 mois pour 95 p. 100 et à 27 et 34

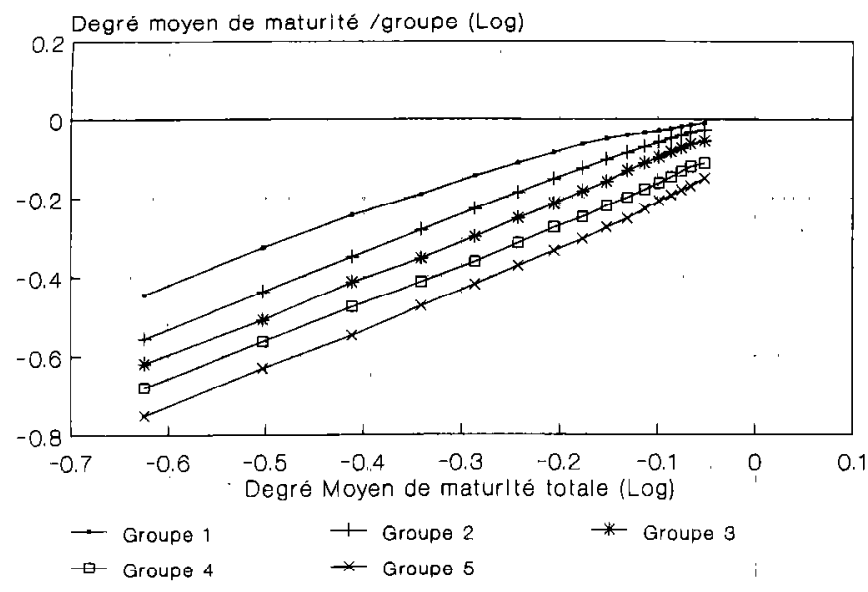

Figure 7 : Courbe allométrique du degré moyen de maturité des différents groupes chez les femelles dans la race Mehraban.

mois pour 98 p. 100 de maturité pondérale chez les femelles et les mâles, elle se réduit à 15 et 18 mois pour 95 p. 100 et à 22 et 25 pour 98 p. 100 de maturité corporelle. Le poids peut-il continuer à augmenter alors que la maturité corporelle est atteinte ? Premièrement, il s'agit de déterminer si les caractères retenus ont tous une incidence sur le poids, lesquels exercent un effet significatif, et quel temps est requis pour qu'ils arrivent à maturité. Les principales sources de variation du poids ont été calculées à cet effet. Deuxièmement, quand un animal arrive à maturité corporelle, un dépôt de graisse commence à se former. Le poids continue donc à augmenter bien que le développement maximal soit atteint. A partir du $15 \mathrm{e}$ mois dans la race Mehraban, la courbe de croissance de la graisse indique une accélération rapide (1).

Parmi les 15 caractères mesurés, les principales sources de variations du poids à tout âge sont la taille au garrot, la longueur du corps, le périmètre abdominal et le périmètre thoracique. L'importance relative de chaque caractère a été calculée en utilisant la régression multiple ajustée par la méthode des moindres carrés pour la part de la variation (variation phénotypique) par le coefficient de détermination $\left(R^{2}\right)$. Les valeurs du coefficient de détermination $\left(R^{2}\right)$ aux âges différents pour chacun des caractères ont été calculées séparément en utilisant la méthode décrite dans la partie Matériel et Méthodes. Les résultats selon les différents âges sont illustrés dans les figures 8 et 9 .

Les résultats montrent que 0,24 , soit 29,15 p. 100 et 0,25 , soit 27,95 p. 100 des variations du poids à l'âge de 3 mois chez les mâles et les femelles respectivement s'expliquent par la taille. Elles diminuent avec l'âge pour atteindre 0,10 , soit 12,78 p. 100 pour les mâles et 0,09 , soit 9,75 p. 100 pour les femelles à 48 mois. En ce qui concerne la longueur du corps, cette variation s'élève à 0,21 , soit 26,21 p. 100 et 0,23 , soit 26,24 p. 100 pour les mâles et les femelles respectivement à l'âge de 3 mois, en diminuant graduellement pour atteindre 0,10 , soit 


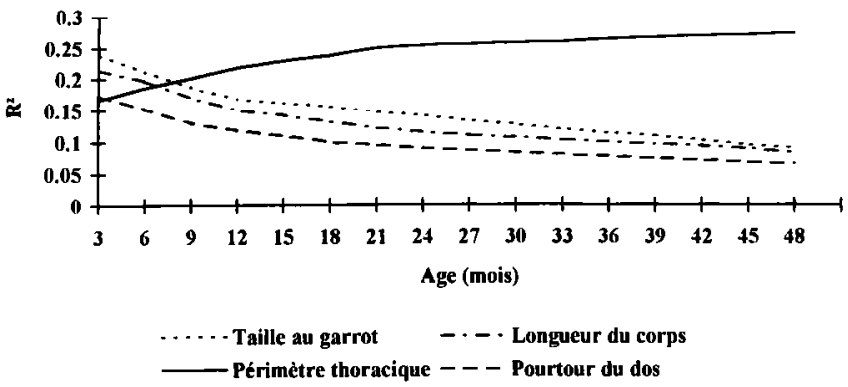

$R^{2}$ total à 3 mois $=082$

$\mathrm{R}^{2}$ total à 48 mols $=0.70$

Figure 8 : Principales sources de variation du poids pour le sexe mâle dans la race Mehraban.

11,71 p. 100 et 0,08 , soit 9,18 p. 100 pour les mâles et les femelles à l'âge de 48 mois. De même, la part de la variation du périmètre abdominal diminue de 0,17 , soit 21,17 p. 100 et 0,18 , soit 20,75 p. 100 à l'âge de 3 mois à 0,08 , soit 9,36 p. 100 et 0,07 , soit 8,03 p. 100 à 48 mois chez les mâles et les femelles respectivement. Par contre, la part de variation due au périmètre thoracique augmente avec l'âge. A l'âge de 3 mois, elle atteint 0,17 , soit 20,35 p. 100 pour les mâles et 0,17 , soit 19,61 p. 100 pour les femelles, tandis qu'elle est de 0,32 , soit $38,66 p$. 100 et 0,27 , soit 31,18 p. 100 à l'âge de 48 mois (fig. 8 , 9). Etant donné l'évolution des variations pondérales en fonction des différents caractères, il apparaît que le meilleur indicateur pour estimer le poids aux différents âges est le périmètre thoracique qui explique le mieux la variation de poids. Or, celui-ci se situe dans le groupe 5 , à maturité tardive. Les caractères repris dans ce groupe arrivent à 95 p. 100 de leur maturité à 28-27 mois chez les mâles et les femelles, et à 98 p. 100 de celle-ci à 35 mois chez les mâles, 33 chez les femelles.

La relation entre le poids et les caractères qui l'influencent significativement (régression multiple du poids sur la taille au garrot, la longueur du corps, le périmètre abdominal et le périmètre thoracique), a été étudiée aux différents âges pour les sexes mâle et femelle. Les résultats de cette analyse sont donnés aux figures $10,11,12$ et 13 .

\section{Corrélations phénotypiques entre le poids et les caractères mesurés}

Les corrélations entre le poids d'une part, la taille au garrot, la longueur du corps et le périmètre abdominal d'autre part diminuent avec l'âge. En revanche, elles augmentent pour le périmètre thoracique (figure 14). La corrélation entre le poids et la taille est de 0,67 pour les mâles et les femelles à l'âge de 3 mois. Elle diminue continuellement jusqu'à l'âge de 48 mois pour atteindre 0,35 . En ce qui concerne la longueur du corps, cette corrélation s'élève à 0,62 à l'âge de 3 mois, en diminuant graduellement pour atteindre 0,30 à l'âge de 48 mois. De même, la corrélation entre le poids et le périmètre abdominal qui est de 0,48 à 3 mois diminue à 0,20 à 48 mois,

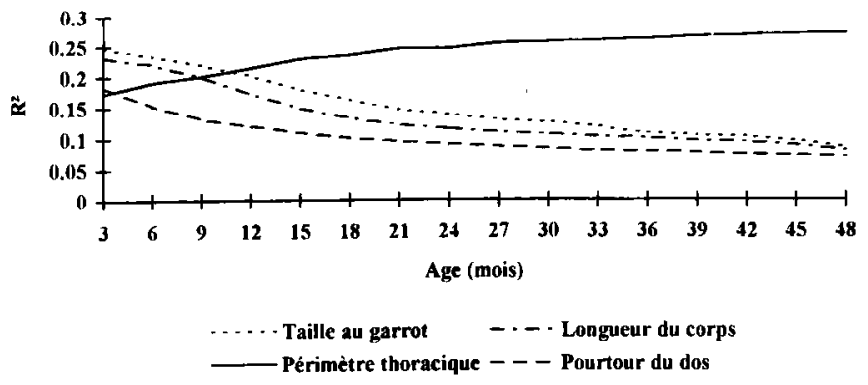

$R^{2}$ total à 3 mois $=0.88$

$R^{2}$ total à 48 mois $=0.87$

Figure 9 : Principales sources de variation du poids pour le sexe femelle dans la race Mehraban.

mais la corrélation entre le poids et le périmètre thoracique augmente avec l'âge. A 3 mois, cette dernière atteint 0,45 pour les femelles, tandis qu'elle est de 0,71 à 48 mois. Cette corrélation élevée, signalée également par Fernandez-Abella (9), permet d'évaluer avec une relative précision le poids de l'animal aux différents âges sans avoir à le peser. II faut noter qu'en matière de corrélation, aucune différence significative $\mathrm{n}$ a été observée entre les deux sexes.

\section{Mesure de la queue grasse}

En Iran, les races ovines présentent un dépôt adipeux caudal (la queue grasse) avec une accumulation importante de graisse qui s'accroît avec le temps. La mesure de la longueur, de la largeur et de la circonférence de cette queue constitue un indicateur important de l'évolution relative de la graisse dont il existe d'autres dépôts, non mesurés dans cette étude. Les trois caractères susmentionnés ont été mesurés aux différents âges (tabl. VI). En ce qui concerne la circonférence de la queue, elle présente une accélération de croissance à partir de 12 mois, chez les deux sexes, mais de façon plus marquée chez les mâles que chez les femelles. Quant à la longueur, la courbe révèle la même évolution, mais à partir de 15 mois. En revanche, sa largeur augmente de 12 à 24 mois puis le gain diminue significativement. Ces résultats ont été comparés avec les courbes établies à partir de l'équation de Brody (fig. 15, 16, 17). Celles-ci indiquent une décroissance constante pour les trois caractères, ce qui est infirmé par les observations empiriques. L'équation de Brody semble donc inadéquate dans ce cas. Une analyse plus approfondie est certes nécessaire, mais elle sortirait du cadre de cette étude.

\section{DISCUSSION}

La courbe de croissance relative permet une surveillance efficace de l'animal mais n'apporte aucun renseignement sur son développement corporel ni sur les modifications morphologiques. Par ailleurs, dans beaucoup de situations, des estimations suffisamment précises du format à 


\section{Poids ( $\mathrm{kg})$}

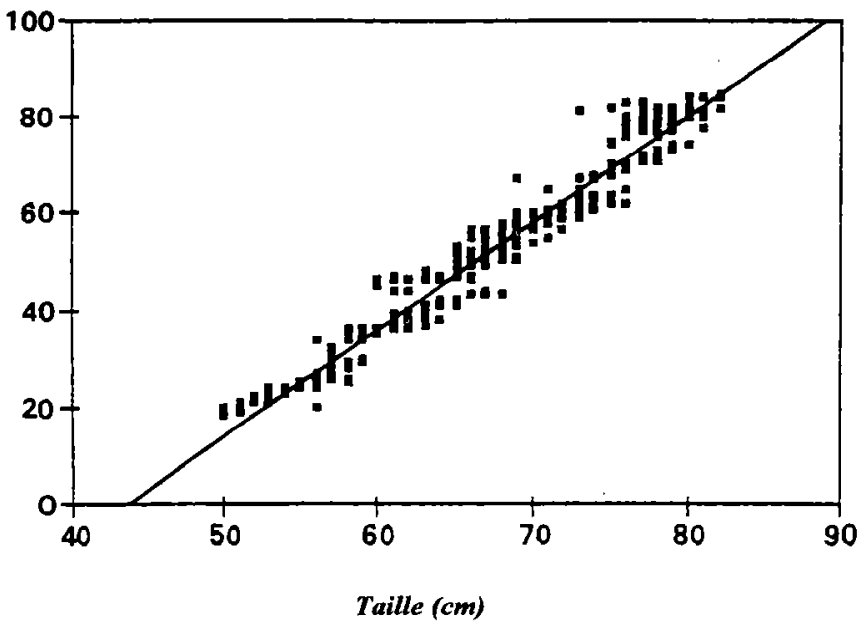

Figure 10 : Relation entre le poids et la taille pour le matériel animal global (male et femelle) dans la race Mehraban.

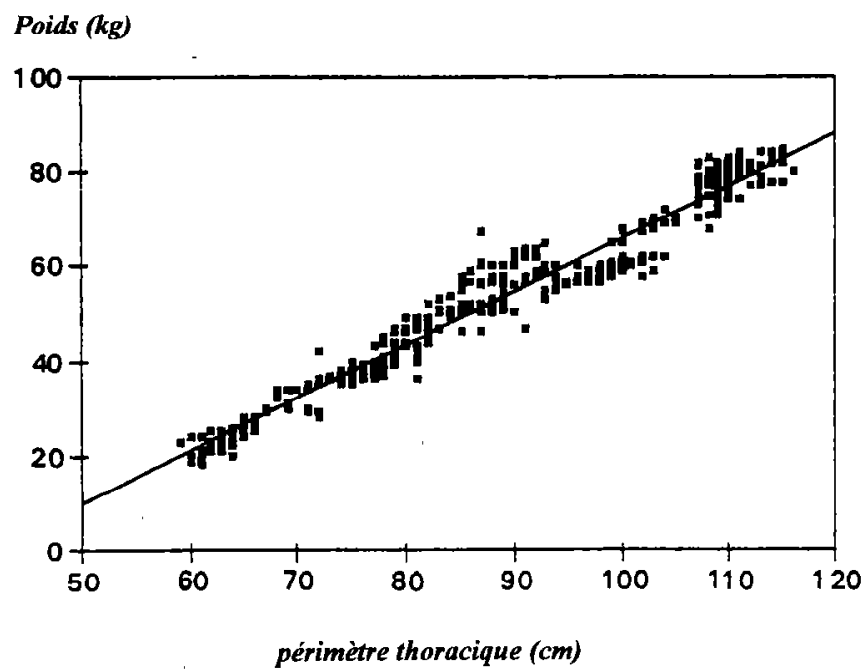

Figure 12 : Relation entre le poids et le périmètre thoracique pour le matériel animal global (mâle et femelle) dans la race Mehraban.

maturité peuvent être obtenues à partir du poids des animaux dont l'alimentation est abondante et qui ont un régime alimentaire constant, ce qui n'est pas toujours facile à réaliser. Si cette condition n'est pas remplie, il faut étudier alors un nombre important de paramètres du développement corporel $(10,26)$. Dans la pratique, le poids est fortement influencé par les facteurs de l'environnement. II était donc intéressant d'étudier le développement corporel et ses relations avec le poids aux différents âges. Cet outil supplémentaire est beaucoup plus précis pour estimer la maturité d'un animal et élaborer un programme de sélection efficace.

Cette étude menée sur 7 années, a montré l'importance des effets du sexe sur tous les caractères mesurés, à tous les âges, tandis que le mode de naissance n'influence que

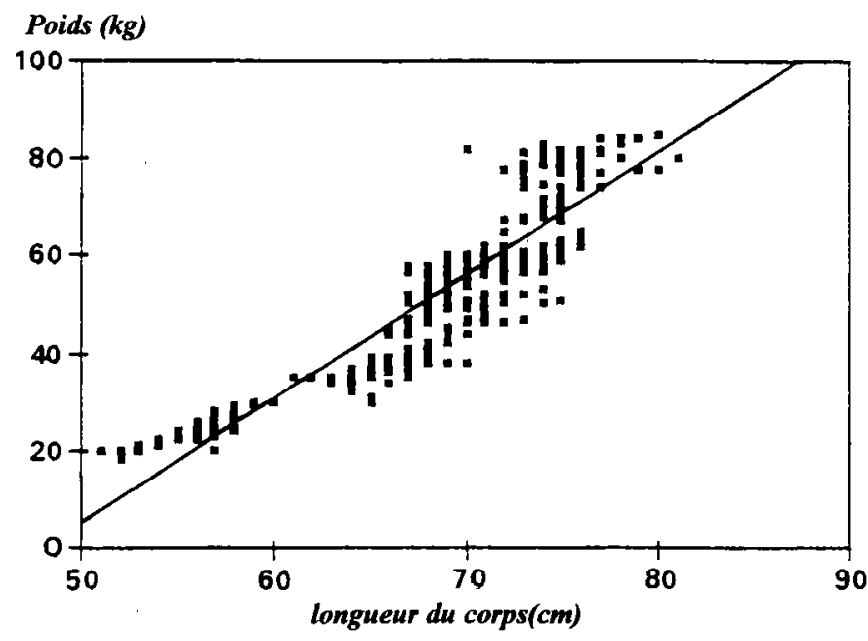

Figure 11 : Relation entre le poids et la longueur du corps pour le matériel animal global (mîlo' et femelle) dans la race Mehraban.

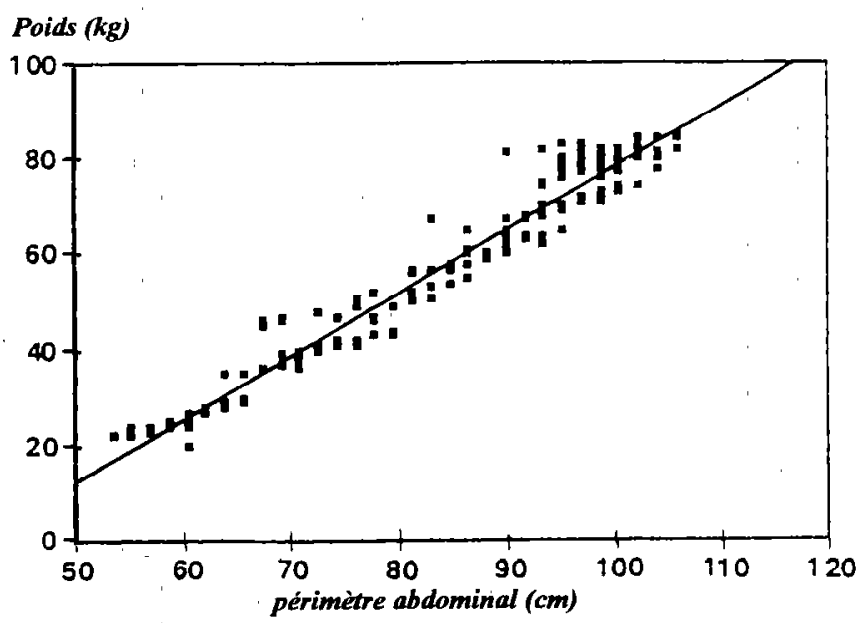

Figure 13 : Relation entre le poids et le périmètre abdominal pour le matériel animal global (mâle et femelle) dans la race Mehraban.

la taille au garrot, la longueur du corps, le pourtour du cou et le périmètre thoracique. L'âge de la mère à l'agnelage est un facteur déterminant sur le poids, la taille et la longueur du corps mais seulement avant sevrage (tabl. I). Ceci peut s'expliquer par le fait que le taux de croissance des agneaux dépend fortement de la production laitière maternelle. Or, l'âge de la brebis influence significativement cette production. II est admis que les agnelles produisent moins de.lait que les brebis adultes. Les agneaux issus des agnelles sont donc plus légers $(3,12,14,17,18$, $20,34)$. Par ailleurs, les agnelles produisent des agneaux plus petits $(1,6)$ que les brebis adultes, mais ils compensent souvent ce retard à l'étape ultérieure de la croissance et la différence devient non significative.

Kazzal (16) a étudié l'effet du sexe, de l'année de nais- 
TABLEAU VI

Développement de la queue grasse $(\mathrm{cm})$ observé et estimé par l'équation de Brody aux différents âges chez les mâles et femelles dans la race Mehraban

\begin{tabular}{|c|c|c|c|c|c|c|c|c|c|c|c|}
\hline \multirow{2}{*}{ Caractère } & \multicolumn{11}{|c|}{ Âge(en mois) } \\
\hline & Naissance & 3 & 6 & 9 & 12 & 15 & 18 & 21 & 24 & 27 & 30 \\
\hline$\frac{\text { Mâle }}{\text { Circonférence }}$ & & & & & & & & & & & \\
\hline $\begin{array}{l}\text { observée } \\
\text { estimée (Brody) }\end{array}$ & $\begin{array}{l}26,05 \\
26,05\end{array}$ & $\begin{array}{l}29,59 \\
31,06\end{array}$ & $\begin{array}{l}32,58 \\
36,01\end{array}$ & $\begin{array}{l}35,09 \\
39,48\end{array}$ & $\begin{array}{l}37,79 \\
42,46\end{array}$ & $\begin{array}{l}41,19 \\
45,02\end{array}$ & $\begin{array}{l}45,99 \\
47,18\end{array}$ & $\begin{array}{l}51,49 \\
49,04\end{array}$ & $\begin{array}{l}57,79 \\
50,64\end{array}$ & $\begin{array}{l}64,29 \\
52,01\end{array}$ & $\begin{array}{l}70,99 \\
53,05\end{array}$ \\
\hline $\begin{array}{l}\text { Longueur } \\
\text { observée } \\
\text { estimée (Brody) }\end{array}$ & $\begin{array}{l}20,65 \\
20,65\end{array}$ & $\begin{array}{l}23,05 \\
24,05\end{array}$ & $\begin{array}{l}25,15 \\
26,50\end{array}$ & $\begin{array}{l}26,95 \\
28,35\end{array}$ & $\begin{array}{l}28,39 \\
29,88\end{array}$ & $\begin{array}{l}29,85 \\
31,23\end{array}$ & $\begin{array}{l}32,35 \\
32,33\end{array}$ & $\begin{array}{l}35,35 \\
33,18\end{array}$ & $\begin{array}{l}39,15 \\
33,88\end{array}$ & $\begin{array}{l}43,45 \\
34,43\end{array}$ & $\begin{array}{l}47,95 \\
34,85\end{array}$ \\
\hline $\begin{array}{l}\text { Largeur } \\
\text { observée } \\
\text { estimée (Brody) }\end{array}$ & $\begin{array}{l}19,21 \\
19,21\end{array}$ & $\begin{array}{l}20,66 \\
23,01\end{array}$ & $\begin{array}{l}21,56 \\
27,01\end{array}$ & $\begin{array}{l}22,36 \\
27,91\end{array}$ & $\begin{array}{l}23,16 \\
28,79\end{array}$ & $\begin{array}{l}24,56 \\
30,19\end{array}$ & $\begin{array}{l}26,86 \\
32,49\end{array}$ & $\begin{array}{l}29,66 \\
35,29\end{array}$ & $\begin{array}{r}32,46 \\
38,09\end{array}$ & $\begin{array}{l}33,96 \\
29,32\end{array}$ & $\begin{array}{l}34,92 \\
30,28\end{array}$ \\
\hline $\begin{array}{l}\text { Femelle } \\
\text { Circonférence }\end{array}$ & & & & & & & & & & & \\
\hline $\begin{array}{l}\text { observée } \\
\text { estimée (Brody) }\end{array}$ & $\begin{array}{l}25,29 \\
25,29\end{array}$ & $\begin{array}{l}28,19 \\
30,49\end{array}$ & $\begin{array}{l}30,66 \\
34,74\end{array}$ & $\begin{array}{l}32,76 \\
38,03\end{array}$ & $\begin{array}{l}34,75 \\
40,85\end{array}$ & $\begin{array}{l}37,46 \\
43,25\end{array}$ & $\begin{array}{l}40,66 \\
45,25\end{array}$ & $\begin{array}{l}44,46 \\
46,92\end{array}$ & $\begin{array}{l}49,26 \\
48,30\end{array}$ & $\begin{array}{l}54,55 \\
49,42\end{array}$ & $\begin{array}{l}60,05 \\
50,35\end{array}$ \\
\hline $\begin{array}{l}\text { Lonqueur } \\
\text { observée } \\
\text { estimée (Brody) }\end{array}$ & $\begin{array}{l}19,77 \\
19,77\end{array}$ & $\begin{array}{l}21,67 \\
22,47\end{array}$ & $\begin{array}{l}23,37 \\
24,47\end{array}$ & $\begin{array}{l}24,87 \\
25,82\end{array}$ & $\begin{array}{l}26,07 \\
26,83\end{array}$ & $\begin{array}{l}27,07 \\
27,70\end{array}$ & $\begin{array}{l}28,57 \\
28,40\end{array}$ & $\begin{array}{l}30,47 \\
28,90\end{array}$ & $\begin{array}{l}33,17 \\
29,25\end{array}$ & $\begin{array}{l}36,37 \\
29,45\end{array}$ & $\begin{array}{l}40,17 \\
29,63\end{array}$ \\
\hline $\begin{array}{l}\text { Largeur } \\
\text { observée } \\
\text { estimée (Brody) }\end{array}$ & $\begin{array}{l}16,94 \\
16,94\end{array}$ & $\begin{array}{l}18,15 \\
20,24\end{array}$ & $\begin{array}{l}19,25 \\
22,64\end{array}$ & $\begin{array}{l}20,15 \\
24,29\end{array}$ & $\begin{array}{l}21,05 \\
25,51\end{array}$ & $\begin{array}{l}22,25 \\
26,28\end{array}$ & $\begin{array}{l}24,25 \\
26,78\end{array}$ & $\begin{array}{l}26,55 \\
27,13\end{array}$ & $\begin{array}{l}29,15 \\
27,35\end{array}$ & $\begin{array}{l}31,16 \\
27,50\end{array}$ & $\begin{array}{l}33,05 \\
27,55\end{array}$ \\
\hline
\end{tabular}

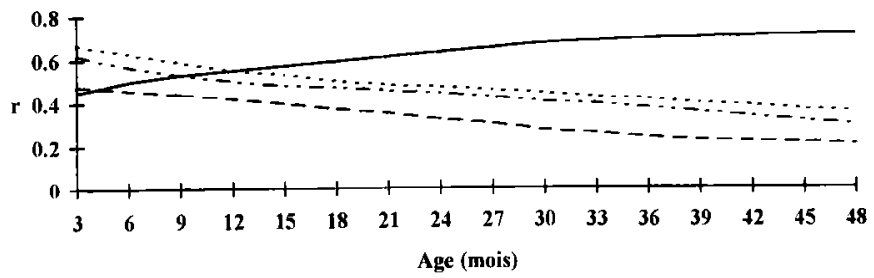

.... - Taille au garrot - - - Longueur du corps

Figure 14 : Corrélations entre le périmètre thoracique, la taille, la longueur du corps, le périmètre abdominal et le poids entre 3 et 48 mois dans la race Mehraban.

sance, de l'âge de la mère et du mois de naissance sur le développement corporel de la race Awassi aux différents âges. Selon lui, les agneaux nés simples sont plus grands pour tous les caractères mesurés que les agneaux de naissance double et l'âge de la mère n'a qu'un effet significatif que sur la taille et la longueur du corps. Wiener et Hayter (33) ont trouvé des effets significatifs du sexe, du mode du naissance et de l'âge de la mère sur les 6 caractères mesurés dans les races Blackface et Cheviot. Juma et al. (15) ont signalé l'effet significatif du mode de naissance sur la taille et la longueur du

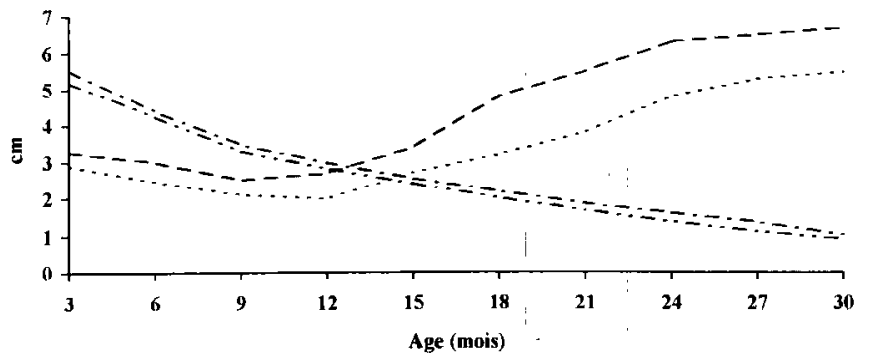

- - - Mâle (observé) ….. . Femelle (observé) - - - Mâle (Brody) - - - Femelle (Brody)

Figure 15 : Croissance relative de la circonférence de la queue grasse de la naissance à 30 mois chez les mâles et femelles dans la race Mehraban.

corps dans la race Awassi. De même, Farid et Makarechian (8) ont signalé l'influence significative des mêmes effets sur la taille, la longueur du corps et le périmètre thoracique dans 5 races iraniennes. Mais, contrairement aux résultats obtenus par Kazzal (16) et Epstein (7), l'année de naissance n'influence pas les caractères étudiés, à l'exception du périmètre thoracique.

Le choix du format optimal pour une étape de croissance ultérieure dans un programme de sélection pour la production de viande, nécessite la connaissance des facteurs économiques et environnementaux ainsi que des 


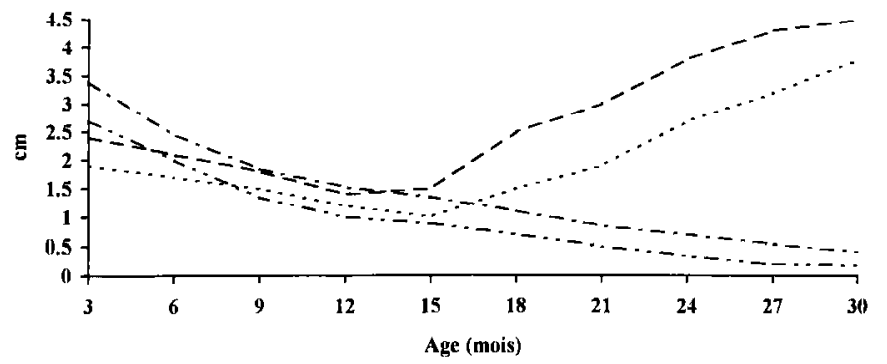

- - Mâle (obsenvé) …. - Femelle (observé) - - - Mâle (Brody) - - - Femelle (Brody)

Figure 16 : Croissance relative de la longueur de la queue grasse de la naissance à 30 mois chez les mâles et femelles dans la race Mehraban.

modes d élevage. Par ailleurs, le temps nécessaire pour atteindre la maturité de certains caractères importants dans la production de viande est un élément économique déterminant dans le choix du format corporel. Les mesures du développement corporel établies pour déterminer le format de maturité finale ont permis d'identifier les caractères précoces et tardifs. La plupart des caractères qui tendent à être plus matures à la naissance ont tendance à se maintenir ainsi jusqu'à la maturité finale. Cette tendance a aussi été signalée par Stobart et al. (28). Les résultats obtenus ne correspondent que partiellement à ceux de Wiener et Hayter (33), étant donné que la race joue un rôle important dans la maturié finale et le temps nécessaire pour l'atteindre. Par exemple, Weiner et Hayter (33) ont étudié la maturité chez les races Cheviot, Blackface, Welsh Mouton, Lincoln et Southdown et ils ont signalé une différence de 4 à 6 mois de la maturité finale dans ces races. La différence en degré de maturité entre les races et entre les sexes a été signalée également par McClelland et al. (19) dans les races Souy, Finish Landrace, Southdown ainsi que par Gaili (11) dans les races à queue grasse Awassi, Najdi et Hejazi. Selon Taylor et al. (29), les races à petit format sont génétiquement plus adéquates sous les climats chauds et secs présentant de grandes variations saisonnières dans les pâturages. Dans ces conditions, elles atteignent plus rapidement la maturité que les grands formats. Ainsi, pour atteindre la productivité optimale à partir du critère du format corporel, il faut : premièrement, choisir le meilleur format à maturité en fonction de l'environnement, du système d'élevage et des facteurs commerciaux, pour un type de production donné ; deuxièmement, dans une population sélectionnée, déterminer les caractères prioritaires en fonction du type de production.

Un autre aspect intéressant est le changement de répartition organique du corps. A l'approche de la maturité, celle-ci se modifie du fait que la proportion de graisse augmente continuellement. Cet effet a été clairement observé ici en ce qui concerne la queue grasse. Une étude précédente concernant le rendement de l'abattage dans la race Mehraban (1), indique également que le poids de la queue grasse augmente avec l'âge, ainsi que son pourcentage par rapport au poids de la carcasse.

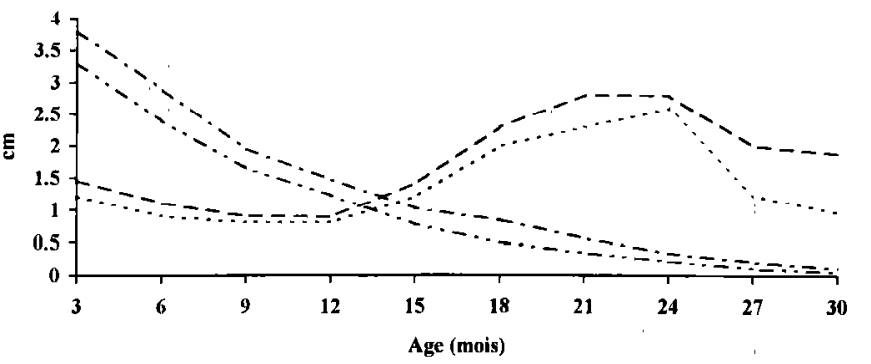

- - Mâle (observé) … . Femelle (observé) - - - Mâle (Brody) - - - Femclle (Brody)

Figure 17 : Croissance relative de la largeur de la queue grasse de la naissance à 30 mois chez les mâles et femelles dans la race Mehraban.

Une proportion importante de la graisse corporelle totale est alors accumulée à cet endroit allant de 40 à 45 p 100. Elle ne contribue pas à valoriser la carcasse. Par ailleurs, est supérieure à celle signalée par Gaili (11) dans les races à queue grasse Awassi (33 p. 100), Najdi et Hejazi (25 p. 100). Le rendement le plus élevé se situe entre 12 et 18 mois et l'accumulation de graisse s'accélère dans cette période. De toute façon, il est préférable d'abattre les animaux vers 12 mois pour éviter un taux de graisse trop élevé.

Un autre élément important dans l'étude du format corporel est l'incidence de l'ingestion alimentaire. Selon Robertson (25), celle-ci augmente jusqu'à sa valeur de maturité bien qu'elle puisse atteindre son maximum avant d'amorcer une légère baisse. En mesurant le rendement (ou efficience) comme le rapport du gain total de poids sur l'ingestion alimentaire totale, on constate que ce rapport baisse de façon continue avec le temps. Par ailleurs, quand un animal est proche de la maturité, il utilise une proportion progressivement plus élevée des aliments ingérés pour maintenir son poids (32). De plus, le niveau d'ingestion alimentaire, l'efficience de la nutrition et par conséquent la croissance de l'animal, sont largement fonction de son format à maturité. Dans les études qui incluent des races de formats différents, le même poids peut refléter des différences significatives dans les degrés de maturité (31). En ce qui concerne l'efficience dans l'ingestion alimentaire selon les sexes, Thompson et al. (32) ont constaté que les brebis maintiennent leur poids à maturité à moindre coût que les béliers. Cet aspect n'a pas été étudié plus précisement pour la race Mehraban.

\section{CONCLUSION}

Pour déterminer le format adulte et l'âge auquel il est atteint, il convient de mesurer des caractères tels que la taille au garrot, qui sont peu influencés par le milieu et dont le rapport avec le poids diminue avec l'âge. Les résultats montrent que la plupart des caractères mesurés évoluent indépendamment des modifications pondérales, ce qui permet de définir de bons indicateurs de croissan- 
ce corporelle. En ce qui concerne le poids à maturité, étant donné que le poids est influencé par de nombreux facteurs, il faut choisir les caractères qui évoluent simultanément à tous les âges. Parmi ceux-ci, le plus fiable est le périmètre thoracique. Or, dans certains modes extensifs d'exploitation, notamment chez les tribus nomades, il est impératif d'obtenir les données indispensables de la façon la plus simple possible. Certaines mesures linéaires comme celle du périmètre thoracique sont à cet égard nettement plus faciles à acquérir que la pesée, tout en étant aussi rigoureuses du point de vue zootechnique.

Enfin, différentes méthodes peuvent être employées pour mesurer la maturité. Celles de Brody (4) ou de Russell (26) semblent bien convenir. Dans le cas où l'allométrie est utilisée pour diminuer les erreurs prévisibles, il faut que l'intervalle entre les mesures soit le plus réduit possible. L'aspect génétique, en ce qui concerne l'héritabilité et les corrélations génétiques entre les caractères mesurés dans le cadre du développement corporel, sera présenté ultérieurement.

\section{Bibliographie}

1. BATHAEI S.S., 1993. Amélioration génétique de la race ovine Mehraban dans le cadre du développement des productions animales en Iran. Thèse doct., Université Libre de Bruxclles, Bruxclles, Bclgique.

2. BATHAEI S.S., LEROY P.L., 1994. Lamb growth performance and factors affecting body weight of Iranian fat tailed Mehraban breed of sheep. Revue Élev. Méd. vét. Pays trop., 47 (1): 113-116.

3. BHAT P.N., KOUL G.L., KOUL S.K., KUMAR R., GARG R.C., 1981 Factors affecting body weight and growth rate of Awassi lambs. J. Agric. Sci. Camb., 97: 449-452.

4. BRODY S., 1945. Bioenergetic and growth. New-York, USA, Reynold Publishing Corporation.

5. CODY R.P., SMITH J.K.,1991. Applied statistics and SAS programming language, 3rd edn. New Jersey, USA, Prentice-Hall Inc.

6. DYRMUNDSSON O.R., 1973. Puberty and early reproductive performance in sheep. I. Ewe lambs. Anim. Breed. Abstr., 41: 273-289.

7. EPSTEIN H., 1985. Awassi sheep. Rome, Italy, FAO. (FAO Animal Production and Health and Paper. No. 57)

8. FARID A., MAKARECHIAN M.,19\% A study on body weight and measurements of some fat-tailed Iranian sheep breeds. I. Some source of variation affecting body weight and measurement of Karakul, Mehraban, Naeini, Ghezel and Bakhtiari ewes. Iran. J. agric. Res. , 5 (1): 55-77.

9. FERNANDEZ-ABELLA D.H., 1986. Genetic and phenotypic correlations and heritabilities of conformations and fleece weight in Polwarth (Ideal) sheep. Archos Zootecnia, 35: 31-40

10. FITZHUNG H,A., 1977. Analysis of growth curves and strategies for altering their shape. J. Anim. Sci., 42: 1036-1051.

11. GNILI E.S.E., 1992. Breed and sex differences in body composition of sheep in relation to maturity and growth rate. J. agric. Sci. Camb., 118: 121-126.

12. GUNN R.G., 1986. A note on the comparative reproductive performance of Friesland x North Country Cheviot and North Country Cheviot ewes on two levels of pasture prior to mating. Anim. Prod., 42: 287-289.

13. HARVEY W.R., 1985. Mixed model least-squares and maximum likelihood computer program. User's guide for LSMLMW. USA, Ohio State University, Department of Dairy Science.

14. JONMUNDSSON J.V., 1977. A sludy of data from the sheep recording associations in Iceland. J. agric. Res. Iceland, 9: 31-42.

15. JUMA K.H., FARAJ M., ELIYA J., AL-AUBAIDY K., 1969. Studics on growth in Awassi sheep. Indian J. Anim. Sci. , 39 (6): 503-512.

16. KAZZAL N.T., 1973. Evaluation of some genetic and environment factors affecting growth and development in Awassi sheep in Iraq. Ph.D. thesis, University of Tennessee, U.S.A.

17. LAND R.B., RUSSELL W.S., DONALD H.P., 1974. The litter size and fertility of Finnish Landrace and Tasmania Merino sheep and their reciprocal crosses. Anim. Prod., 18: 265-271.

18. MAKARECHIAN M., FARID A., SEFIDBAKHT N., 1982. Crosshreeding of Iranian fat-tailed sheep. VI. Reproductive performance and lamb production in Karakul, Mehraban and Naeini breeds. Iran. agric. Res., 1 (1): 1-15.

19. McCLELLAND T.H., BONAITI B., TAYLOR C.S., 1976. Breed dif ferences in body composition of equally mature sheep. Anim. Prod. , 23 281-293.

20. NÄSHOLM A., DANELL Ö., 1990. Growth and mature weight of Swedish Finewool Landrace ewes. Acta Agric. scand., 40: 71-81.

21. PRUD'HON M., 1976. Croissance, engraissement et qualité des carcasses d'agneaux et de chevreaux. La croissance globale de l'agneau : ses caractéristiques et ses lois. In : 2e journées de la recherche ovine et caprine, INRA, ITOVIC, 1er-2 décembre 1976. Paris, France, ITOVIC-SPEOC, p. 6-26.

22. PRUD'HON M., 1985. Phénomènes de croissance. Revue Elev. ovin, 329:17-20.

23. REISS M.J., 1989. The allometry of growth and reproduction. Cambridge University Press.

24. RICORDEAU G., BOCCARD R., 1961. Relations entre la quantité de lait consommé par les agneaux et leur croissance. Annls Zootech., 10 (2) : 113-125.

25. ROBFRTSON A., 1980. Genetic aspects of growth. In: World Cong. on sheep and beef cattle breeding in New Zeland, Massey University, New Zealand, Nov. 3-5 1980 , p. 427-437.

26. RUSSELL W.S., 1975. The growth of Ayrshire cattle: an analysis of linear body measurements. Anim. Prod., 21: 217-226.

27. SAS Institute Inc., 1985. SAS User's guide; Statistics. Cary, USA, SAS Institute Inc.

28. STOBART R.H. BASSETT J.W. CARTWRIGHT T.C., BLACKWELL R.L., 1986. An analysis of body weights and maturing patterns in western range ewes. J. Anim. Sci., 63 (3): 729-740.

29. TAYLOR C.R., CALDWELL S.L., ROWNTREE V.J., 1972. Running up and down hills: some consequences of size. Science N.Y., 178: 1096-1097.

30. TAYLOR C.S., 1980. Genetically standardized growth equations. Anim. Prod., 30:167-175.

31. THOMPSON J.M., PARKS J.R,, 1983. Food intake, growth and body composition in Australian Merino and Dorset Horn sheep. Anim. Prod., 36: $471-479$.

32. THOMPSON J.M., PARKS J.R., PERRY D., 1985. Food intake, grow th and body composition in Australian Merino sheep selected for hight and low weaning weight. 1. Food intake, food efficiency and growth. Anim. Prod., 40: 55-70. 


\section{S.S. Bathaei}

33. WIENER G., HAY IEK S., 1974. Body size and conformation in sheep from birth to maturity as affected by breed, crossbreeding, maternal and other factors. Anim. Prod., 19: 47-65.

BATHAEI (S.S.). Growth and body development from birth to maturity in the Mehraban Iranian fat-tailcd shccp. Revue Élev. Méd. vét. Pays trop., 1995, 48 (2) : 181-194

The growth of 15 linear body measurements in Mehraban Iranian fat-tailed sheep was analysed by least-squares method. Between 1984 and 1990, measurements concerning 1,238 Mehraban sheep were taken at 3-monthly intervals from 3 to 48 months of age. The effects of sex, type of birth, year of birth and age of dam on body weight and 15 measurements were studied. Only sex significantly influenced all the hody measurements at different ages. Frowth curves were established using the mean values at 16 different ages. The estimated degrees of maturity at $98 \%$ were 25 and 22 months for males and females, respectively. The application of allometry to the measurements is examined as means of plotting the curves with greater accuracy. Among the 15 characteristics measured, wither height, body length, back circumference and chest circumference were the most significant sources of weight variation, while chest circumference was the best indicator of the weight variations at different ages. The other factors evolve almost independently of weight.

Key words: Mehraban sheep - Growth - Weight gain - Maturity - Body measurement - Iran.
34. WRIGHT L.A., THRIFT F.A., DU'I'T R.H., 1975. Influence of ewe age on productive characters of Southdown sheep. J. Anim. Sci., 41 (2): 517 521.

BATHAEI (S.S.).Crecimiento y desarrollo corporal del nacimiento a la maduré $<$ en la raza iraní de ovinus Mehraban. Revue Élev. Méd. vét. Pays trop., 1995, 48 (2) : 181-194

El crecimiento de 15 medidas corporalcs lincares en la raza iraní de ovinos Mehraban, fue analizado mediante el método de la regresion. Se tomaron a intervalos de 3 meses las medidas correspondientes a 1238 animales, de 3 a 48 meses de edad, entre 1984 y 1990. Se estudiaron los efectos del sexo, del tipo de parto, del año de nacimiento y de la edad de la madre sobrez el peso, así como sobre las 15 características medidas. El sexo es el único factor que presenta una influencia sobre todos los caractéres, a cualquier edad. Las curvas de crecimiento se establecieron con precisión en base a los valores promedio a 16 edades diferentes. La estimación a 98 p. 100 del grado de madurez es de 25 a 22 meses para machos y hembras respectivamente. La aplicación de la alometría a las características medidas se estudió con el objeto de establecer curvas de crecimiento más exactas. La latura a la cruz y la longitud corporal, así como los perímetros abdominal y torácico, representan mejor las variaciones del peso. Entre estas características, el perímetro torácico es el mejor indicador de estas variaciones a las diferentes edades. En cuanto a los otros factores, evolucionan de manera casi inependiente al peso.

Palabras clave : Ovino Mehraban - Crecimiento - Ganancia de peso Madurez - Medición del cuerpo - Irán. 